\title{
VALIDADE E CONFIABILIDADE DO MORAL DISTRESS SCALE ADAPTADO EM UMA AMOSTRA DE ENFERMEIROS
}

Julia Zancan Bresolin¹, Graziele de Lima Dalmolin², Isolina Maria Alberto Fruet ${ }^{3}$, Tania Solange Bosi de Souza Magnago ${ }^{4}$, Amanda Ceschini Rigue ${ }^{5}$

Objetivo: buscou-se analisar a validade e confiabilidade do Moral Distress Scale (MDS), adaptado em amostra de enfermeiros de uma instituição hospitalar. Metodologia: trata-se de uma pesquisa de abordagem quantitativa realizada com 144 enfermeiros de instituição hospitalar da região central do Rio Grande do Sul, através da aplicação do MDS adaptado, validado e normatizado para uso no Brasil. Para análise dos dados, utilizou-se análise fatorial, alfa de Cronbach e estatística descritiva. Resultados: a partir da análise, foi realizado o agrupamento das questões validadas em três fatores associados ao sofrimento moral: falta de competência na equipe de trabalho, negação do papel da Enfermagem como advogada do paciente e condições de trabalho insuficientes. Conclusão: o instrumento utilizado na pesquisa mostrou-se válido e fidedigno na realidade estudada.

Descritores: Enfermagem, Moral, Estresse Psicológico, Ética em Enfermagem.

\section{VALIDITY AND RELIABILITY OF THE ADAPTED MORAL DISTRESS SCALE IN A SAMPLE OF NURSES}

Objective: the study aimed to analyze the validity and reliability of the adapted Moral Distress Scale (MDS) in a sample of nurses from a hospital. Methodology: it is a cross-sectional study conducted with 144 hospital nurses from the central region of Rio Grande do Sul, through the application of the MDS adapted, validated and standardized for use in Brazil. For data analysis we used factor analysis, Cronbach alpha and descriptive statistics. Results: the factorial analysis of the instrument allowed the grouping of the issues validated in three dimensions associated with moral suffering: staff's lack of competence; the nursing denial of their role as patient advocacy; inadequate work conditions. Conclusion: the instrument used in the study proved to be valid and reliable in the studied reality.

Descriptors: Nursing, Morale, Stress Psychological. Ethics, Nursing.

\section{VALIDEZ Y CONFIABILIDAD DEL MORAL DISTRESS SCALE ADAPTADO EN MUESTRA DE ENFERMEROS}

Objetivo: el estudio buscó analizar la validez y confiabilidad del Moral Distress Scale (MDS) adaptado en una muestra de enfermeros de una institución hospitalaria. Mertodologia: se trata de un estudio transversal realizado con 144 enfermeros de institución hospitalaria de la región central del Río Grande del Sur, a través de la aplicación del MDS adaptado, validado y normatizado para el uso en Brasil. Para el análisis de los datos, se utilizó el análisis factorial, alfa de Cronbach y estadística descriptiva. Resultados: El análisis factorial del instrumento permitió la agrupación de las cuestiones validadas en tres dimensiones asociadas al sufrimiento moral: falta de competencia del equipo de trabajo; negación del papel de la enfermería como abogada del paciente; conditiones de trabajo insuficientes. Conclusión: el instrumento utilizado en la investigación se mostró válido y fidedigno en la realidad estudiada.

Descriptores: EEnfermería, Moral, Estrés Psicológico, Ética en Enfermería.

${ }^{1}$ Enfermeira. Mestranda em Enfermagem do Programa de Pós-Graduação em Enfermagem da Universidade Federal de Santa Maria (PPGEnf/UFSM). ${ }^{2}$ Enfermeira. Doutora em Enfermagem. Professora Adjunta do Departamento de Enfermagem. Universidade Federal de Santa Maria, Santa Maria, RS, Brasil. Email grazi.dalmolin@gmail.com

${ }^{3}$ Enfermeira. Mestranda em Enfermagem do PPGEnf/UFSM

${ }^{4}$ Doutora em Enfermagem. Professora Adjunta do DENFE/UFSM.

${ }^{5}$ Enfermeira. Residente do Programa de Residência Multiprofissional da UFSM - Hospital Universitário de Santa Maria (HUSM). 


\section{INTRODUÇÃO}

A Enfermagem, na sua rotina de trabalho, pode deparar-se com uma variedade de situações geradoras de conflitos éticos e dilemas morais. Essas situações são fontes de sofrimento moral (SM), ocorrendo quando os profissionais reconhecem a maneira mais correta de agir, porém parecem não se sentir seguros e apoiados para agir conforme seus conhecimentos, valores e crenças. (1)

O SM foi conceituado há mais de 30 anos. Originalmente, foi considerado como um doloroso desequilíbrio psicológico resultante de situações em que o profissional de saúde reconhece a conduta ética apropriada a ser seguida, porém, por pressões institucionais torna-se, praticamente impossivel ou incapaz de prosseguir com o curso de ação considerado correto, seja por obstáculos como falta de tempo, relutância da supervisão, estrutura inibidora do poder médico, políticas institucionais ou considerações legais.(2)

Sentimentos de impotência, frustração, raiva e tristeza são os efeitos biopsicossociais mais comuns resultantes do SM. A introspecção também é uma das manifestações apresentadas por profissionais de enfermagem que recebem pouco ou nenhum apoio durante o enfrentamento dos conflitos morais. Essas manifestações podem acarretar respostas emocionais, como descontentamento com o trabalho, relutância em ir trabalhar ou até mesmo abandono da profissão. ${ }^{(3)}$

O instrumento mais utilizado para mensurar SM em profissionais de Enfermagem é o Moral Distress Scale (MDS), criado em 1995 e ajustado, em 2001, quando aplicado em 214 enfermeiros atuantes em hospitais norte-americanos. (4) No Brasil, foi validado em 2009, e adaptado em 2012, constituindo-se numa ferramenta disponivel para avaliação do SM neste contexto.(5)

Assim, esse estudo justifica-se pela necessidade de avaliação do SM a partir da ocorrência de dilemas éticos e morais vivenciados por enfermeiros. Considera-se relevante o desenvolvimento dessa pesquisa para a construção do conhecimento da Enfermagem, para reflexão dos profissionais no que se refere às situações conflituosas que resultam em SM e para testar a fidedignidade do MDS adaptado.

Desse modo, apresentou-se como objetivo analisar a validade e confiabilidade do MDS adaptado em uma amostra de enfermeiros de uma instituição hospitalar.

\section{METODOLOGIA}

Trata-se de um estudo transversal, realizado em um hospital universitário, localizado em um município da região central do Rio Grande do Sul. Os participantes do estudo foram enfermeiros que apresentaram interesse em responder o questionário e que tivessem, no mínimo, um mês de atuação. O critério de exclusão dos participantes limitou- se a não estar no ambiente de trabalho no período de coleta de dados por afastamentos ou férias.

O instrumento utilizado para coleta de dados foi o MDS adaptado, juntamente a um componente de caracterização sociodemográfica. O MDS foi originalmente desenvolvido em contexto norte americano ${ }^{(6)}$, sendo submetido ao processo de tradução reversa, adaptação cultural e validação(5). Percebeuse que situações identificadas na realidade brasileira, fontes de SM, como questões organizacionais de insuficiência de profissionais, precariedade de recursos materiais, e falta de autonomia profissional não estavam contempladas, sendo adaptado para considerar essas particularidades ${ }^{(5)}$. O MDS adaptado compõe-se de 39 questões, em escala Likert de sete pontos, analisando intensidade e frequência de SM. A escala varia de zero (0) para nunca ocorrer ou nenhuma frequência, a seis (6) para SM muito intenso ou muito frequente.

Utilizou-se amostra não-probabilistica por conveniência. Entretanto, para reduzir a ocorrência de possiveis vieses, adotou-se o critério de seleção do tamanho amostral(7) que estimou o número mínimo de 144 participantes, tomando-se por base a população de 228 enfermeiros.

A coleta de dados foi realizada no período de dezembro/2014 a março/2015, quando os enfermeiros foram apresentados aos objetivos e forma de participação no estudo, agendando-se um horário em que o enfermeiro estivesse disponível para responder o questionário, ou foi entregue e agendado seu retorno. Realizaram-se até três tentativas de busca.

Para análise, utilizaram-se análise fatorial, alfa de Cronbach, estatística descritiva, com distribuição de frequência, medidas de posição e medidas de dispersão.

Para inclusão dos dados no processo de análise da pesquisa, foi utilizado o programa Epi-info $₫$, versão 6.4, com dupla digitação independente. Posteriormente, para análise dos dados, foi utilizado o programa Predictive Analytics Software, da SPSS Inc., Chicago, USA (PASW Statistic ${ }^{\circledR}$ ) versão 18.0 para Windows.

Foram respeitados os preceitos éticos da pesquisa conforme Resolução 466/12, tendo recebido parecer favorável do Comitê de Ética em Pesquisa local através do Certificado de Apresentação para Apreciação Ética (CAAE) número 24330213.8.0000.5346.

\section{RESULTADOS}

Dos 228 enfermeiros convidados a participar do estudo, obteve-se um total de retorno de 144 enfermeiros, porém, no processo de análise, excluíram-se 17 instrumentos por apresentarem incorreção na marcação das questões, ou estarem em branco, sendo a amostra constituída por 127 participantes. 
Na tabela 1, apresentam-se os dados sociodemográficos dos participantes do estudo.

Tabela 1 - Dados sociodemográficos dos enfermeiros participantes do estudo.

\begin{tabular}{|c|c|c|}
\hline Dados sociodemográficos & & \\
\hline Faixa etária (anos) & $\mathbf{N}$ & $\%$ \\
\hline$<25$ & $\mathrm{Ol}$ & 0,8 \\
\hline $25-34$ & 43 & 33,86 \\
\hline $35-44$ & 46 & 36,2 \\
\hline $45-54$ & 26 & 20,4 \\
\hline $55-65$ & 11 & 8,7 \\
\hline Sexo & $\mathbf{N}$ & $\%$ \\
\hline Feminino & 114 & 89,8 \\
\hline Masculino & 13 & 10,2 \\
\hline Escolaridade & $\mathbf{N}$ & $\%$ \\
\hline Graduação & 11 & 8,7 \\
\hline Especialização & 71 & 55,9 \\
\hline Mestrado & 39 & 30,7 \\
\hline Doutorado & 6 & 4,7 \\
\hline Tempo de serviço (anos) & $\mathbf{N}$ & $\%$ \\
\hline$<1$ & 33 & 26 \\
\hline $1-11$ & 57 & 44,9 \\
\hline $12-24$ & 24 & 18,9 \\
\hline$>25$ & 11 & 8,6 \\
\hline Não informaram & 02 & 1,6 \\
\hline Unidade de trabalho & $\mathbf{N}$ & $\%$ \\
\hline Materno Infantil & 14 & 11 \\
\hline UTIs & 21 & 16,5 \\
\hline Bloco Cirúrgico & 16 & 12,6 \\
\hline Pronto Socorro & 18 & 14,2 \\
\hline Clínicas de internação & 18 & 14,2 \\
\hline Ambulatórios & 17 & 13,4 \\
\hline Administrativas & 06 & 4,7 \\
\hline Hemato Oncologia & 12 & 9,4 \\
\hline Psiquiatria & 03 & 2,4 \\
\hline Não informaram & 02 & 1,6 \\
\hline
\end{tabular}

Para análise da validade, inicialmente, ocorreu a submissão das 39 questões do instrumento à análise fatorial exploratória (em blocos). Realizou-se análise de componentes principais com aplicação de rotação ortogonal Varimax, com objetivo de maximizar as altas correlações e minimizar as baixas, indicando a melhor adequação das variáveis aos componentes identificados ${ }^{(8)}$. Os critérios para formação dos fatores foram o grau de associação e de subjetividade entre as variáveis.

Para facilitar o agrupamento das questões, realizou-se o processo de exclusão gradual de cada uma daquelas questões que apresentavam correlações baixas em seus blocos (baixa carga fatorial, inferior a 0,40) e aquelas que não apresentavam coerência conceitual com os fatores formados. Ao final dessa análise, foram excluídas 15 questões, sendo validadas 24 questões em três fatores.

A avaliação da fidedignidade do instrumento foi realizada através do cálculo do alfa de Cronbach, que apresentou valor 0,973 , enquanto que os coeficientes dos fatores situaramse entre 0,738 e 0,978, comprovando sua fidedignidade. Os fatores definidos receberam as seguintes denominações: falta de competência na equipe de trabalho, negação do papel do enfermeiro como advogado do paciente e condições de trabalho insuficientes.

Falta de competência na equipe de trabalho relacionase à insegurança dos profissionais de Enfermagem em atuar junto com outros profissionais da equipe de Enfermagem, saúde e serviços de apoio, que não thes inspiram confiança em suas ações, conforme suas escolhas, valores e convicções ${ }^{(9)}$. Negação do papel da enfermagem como aduogada do paciente como potencial não utilizado pelos profissionais de enfermagem para reivindicar os direitos dos pacientes ${ }^{(10)}$. As condições de trabalho insuficientes como fonte de SM relacionam-se à organização do trabalho, associadas à carência de recursos materiais e humanos ${ }^{(11)}$.

Os três fatores propostos explicaram $77,26 \%$ da variação das questões originais, representando um grau adequado de sintetização dos dados, facilitando seu manuseio e interpretação.

A Tabela 2 sintetiza os resultados da análise de componentes principais conduzida, com rotação ortogonal (Varimax), apresentando as cargas fatoriais de cada questão. A medida de Kaiser-Meyer-Olkin verificou a adequação amostral para a análise $(\mathrm{KMO}=0,938)$. O teste de esfericidade de Bartlett [qui-quadrado $(276)=3697,186$ p menor que 0,001] indicou que as correlações entre os itens são suficientes para a realização da análise fatorial. 
Tabela 2 - Análise Fatorial Exploratória (rotação Varimax)

\begin{tabular}{|c|c|c|c|c|}
\hline Falta de competência na equipe de trabalho & Bloco & F1 & F2 & F3 \\
\hline $\begin{array}{l}\text { q24:Trabalhar com enfermeiras que não possuem a competência necessária que a } \\
\text { condição do paciente requer. }\end{array}$ & 0,803 & 0,792 & 0,315 & 0,275 \\
\hline $\begin{array}{l}\text { q25: Trabalhar com técnicos/auxiliares de enfermagem que não possuem a } \\
\text { competência necessária que a condição do paciente requer. }\end{array}$ & 0,824 & 0,804 & 0,277 & 0,318 \\
\hline $\begin{array}{l}\text { q26: Trabalhar com estudantes de Medicina ou Enfermagem que não possuem a } \\
\text { competência necessária que a condição do paciente requer. }\end{array}$ & 0,789 & 0,869 & 0,325 & 0,171 \\
\hline $\begin{array}{l}\text { q27: Trabalhar com médicos que não possuem a competência necessária que a } \\
\text { condição do paciente requer. }\end{array}$ & 0,889 & 0,869 & 0,325 & 0,171 \\
\hline $\begin{array}{l}\text { q28: Trabalhar com serviços de apoio que não possuem a competência necessária } \\
\text { que a condição do paciente requer. }\end{array}$ & 0,857 & 0,853 & 0,346 & 0,094 \\
\hline q29: Trabalhar com enfermeiras que não possuem competência para atuar. & 0,885 & 0,854 & 0,285 & 0,274 \\
\hline q30: Trabalhar com técnicos/auxiliares que não possuem competência para atuar. & 0,903 & 0,846 & 0,333 & 0,277 \\
\hline q31: Trabalhar com médicos que não possuem competência para atuar. & 0,868 & 0,830 & 0,363 & 0,218 \\
\hline q32: Trabalhar com serviços de apoio que não possuem competência para atuar. & 0,795 & 0,775 & 0,417 & 0,145 \\
\hline q33:Trabalhar com estudantes de medicina ou enfermagem. & 0,823 & 0,814 & 0,366 & 0,164 \\
\hline Negação do papel da enfermagem como advogada do paciente & Bloco & Fl & F2 & F3 \\
\hline $\begin{array}{l}\text { q9: Evitar tomar providência ao constatar que um membro de enfermagem aplica a } \\
\text { medicação equivocada e deixa de reportá-lo. }\end{array}$ & 0,668 & 0,459 & 0,532 & 0,418 \\
\hline $\begin{array}{l}\text { q10: Permitir que estudantes de medicina realizem procedimentos dolorosos em } \\
\text { pacientes apenas para aprimorar suas habilidades. }\end{array}$ & 0,710 & 0,489 & 0,591 & 0,349 \\
\hline $\begin{array}{l}\text { q16: Observar, sem tomar providências, quando a equipe de enfermagem não } \\
\text { respeita a privacidade do paciente. }\end{array}$ & 0,690 & 0,368 & 0,586 & 0,460 \\
\hline $\begin{array}{l}\text { q17: Obedecer a ordem médica de não dizer a verdade ao paciente, mesmo quando } \\
\text { o paciente lhe pede a verdade. }\end{array}$ & 0,757 & 0,326 & 0,742 & 0,317 \\
\hline $\begin{array}{l}\text { q18: Prestar auxilio a um médico que, em sua opinião, está agindo de forma } \\
\text { incompetente para com o paciente. }\end{array}$ & 0,714 & 0,406 & 0,649 & 0,357 \\
\hline $\begin{array}{l}\text { q21: Aceitar o pedido do médico de não discutir, com o paciente, sua reanimação } \\
\text { em caso de parada cardíaca. }\end{array}$ & 0,696 & 0,237 & 0,746 & 0,290 \\
\hline $\begin{array}{l}\text { q23: Acatar o pedido do médico para não falar sobre morte com um paciente } \\
\text { moribundo que the pergunta sobre morrer. }\end{array}$ & 0,754 & 0,217 & 0,812 & 0,219 \\
\hline $\begin{array}{l}\text { q34: Evitar tomar providências em situações de morte de pacientes associada à } \\
\text { negligência profissional. }\end{array}$ & 0,799 & 0,432 & 0,768 & 0,150 \\
\hline $\begin{array}{l}\text { q37: Dar início a procedimentos intensivos para salvar a vida quando o paciente } \\
\text { terminal já manifestou o desejo de morrer. }\end{array}$ & 0,738 & 0,298 & 0,804 & 0,048 \\
\hline $\begin{array}{l}\text { q38: Evitar tomar providência quando percebe o abandono do paciente terminal } \\
\text { pela equipe de saúde. }\end{array}$ & 0,843 & 0,398 & 0,810 & 0,169 \\
\hline $\begin{array}{l}\text { q39: Evitar tomar providência quando percebe o abandono do paciente terminal } \\
\text { pela familia. }\end{array}$ & 0,821 & 0,385 & 0,806 & 0,150 \\
\hline Condições de trabalho insuficientes & Bloco & Fl & F2 & F3 \\
\hline $\begin{array}{l}\text { q01: Fazer improvisações para enfrentar a falta de material no cuidado aos } \\
\text { pacientes. }\end{array}$ & 0,400 & 0,123 & 0,063 & 0,617 \\
\hline $\begin{array}{l}\text { q14: Necessitar priorizar os pacientes a serem cuidados pela insuficiência de } \\
\text { recursos humanos. }\end{array}$ & 0,801 & 0,282 & 0,324 & 0,785 \\
\hline $\begin{array}{l}\text { q15: Necessitar delegar cuidados de enfermagem aos familiares dos pacientes pela } \\
\text { insuficiência de recursos humanos. }\end{array}$ & 0,714 & 0,206 & 0,365 & 0,734 \\
\hline
\end{tabular}


As questões com maior carga fatorial no primeiro fator foram a "q26: trabalhar com estudantes de Medicina ou Enfermagem que não possuem a competência necessária que a condição do paciente requer" e a "q27: trabalhar com médicos que não possuem a competência necessária que a condição do paciente requer"; no segundo fator, foi a "q23: acatar o pedido do médico para não falar sobre morte com um paciente moribundo que the pergunta sobre morrer"; e, no terceiro fator, foi a "ql4: necessitar priorizar os pacientes a serem cuidados pela insuficiência de recursos humanos".

$\mathrm{Na}$ Tabela 3, apresenta-se a média, mediana, desviopadrão, o valor do alfa de Crombach e os valores de correlação para cada fator.

Tabela 3 - Média, mediana, desvio padrão, alfa de Cronbach e valores de correlação para os três fatores de sofrimento moral.

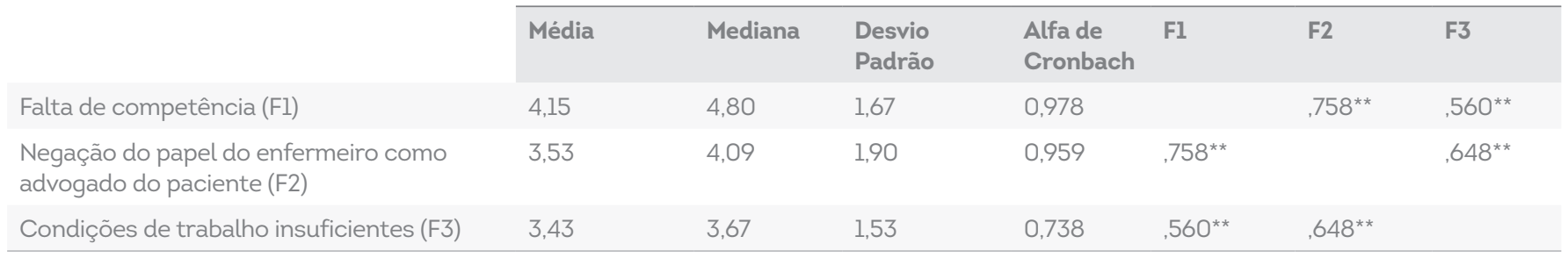

${ }^{* *} \mathrm{p}<0,001$

Esses resultados obtidos a partir do estudo da validade de constructo do MDS confirmam que seus itens medem o SM por meio dos fatores designados teoricamente, constituindose num instrumento adequado e fidedigno na avaliação do SM em enfermeiros.

\section{DISCUSSÃO}

O SM constitui-se num desafio diante de situações problemáticas, quando o profissional tem um julgamento ético ou moral sobre a situação, mas diferente da percepção de outros profissionais também responsáveis pelo cuidado(12). Dessa forma, faz-se importante a avaliação do SM entre os profissionais de enfermagem, mediante instrumentos específicos como o MDS, e sua versão adaptada para o contexto brasileiro

Numa primeira aplicação do MDS adaptado, a amostra foi composta por 247 profissionais de enfermagem que resultou em 23 questões validadadas, cinco fatores, e valor do alfa de Cronbach de 0,95(13). Em outro estudo, com o mesmo instrumento, a amostra foi constituída por 334 profissionais de enfermagem, quatro fatores, 20 questões validadas e alfa de Cronbach de 0,93(14). No atual estudo, com 127 enfermeiros, apresentou-se três fatores, 24 questões validadas e alfa de 0,97 .

Ao comparar os que utilizaram o MDS adaptado, percebe-se que, neste estudo, apesar do menor número de fatores, há o maior número de questões validadas do instrumento, apresentando também o maior valor de alfa $(0,97)$, indicando um alto nivel de consistência interna. Salienta-se que os fatores encontrados, no presente estudo, receberam as mesmas denominações dos estudos realizados anteriormente $e^{(13.14)}$, porém com número de questões diferentes, pois compreendeu-se que a conceituação adotada pelos estudos reflete o conteúdo representado pelas questões do instrumento.

Destaca-se que as questões que constituem o fator "falta de competência na equipe de trabalho" apresentaram as maiores cargas fatoriais, nos três estudos, demonstrando uma elevada aderência e relação com o SM. Os valores da média $(4,15)$ e mediana $(4,80)$, encontradas na avaliação desse fator, a constituem na variável com maior percepção de SM entre os enfermeiros, caracterizando SM de moderado a intenso, considerando o intervalo de zero à seis (0-6) da escala.

Em relação aos demais fatores, pode-se associar a negação do papel da enfermagem como advogada do paciente e condições de trabalho insuficientes. A prática profissional é dificultada pela falta de recursos, conflitos profissionais e culturas organizacionais não encorajadoras ao desempenho da advocacia do enfermeiro que, muitas vezes, também tem como desafio o despreparo educacional e a dificuldade de raciocínio ético, situações potencialmente não trabalhadas nas instituições, causando constrangimentos ${ }^{(15)}$.

Diante disso, os dados desse estudo vão ao encontro de outros que também investigaram o SM em diferentes contextos do cuidado à saúde, considerando que os valores das médias e medianas apresentadas dos fatores estão todas num nível de moderado a intenso, haja vista o intervalo de 0 a 6 . O SM, como definido pelos fatores apresentados, está ligado às situações em que os enfermeiros não têm possibilidades de implementar uma escolha ética e moral, apresentando como consequência o interesse por parte desses profissionais em deixar seus cargos atuais na Enfermagem ${ }^{(16)}$.

A aplicação do MDS adaptado é uma estratégia condizente com o momento atual da Enfermagem. Faz-se necessária a identificação da vivência do SM, para o conhecimento dessa 
realidade e busca de elementos que venham a contribuir para diminuir as situações que provocam esse fenômeno, como o incentivo à agência moral e manutenção da integridade moral, garantindo um cuidado adequado e de qualidade ${ }^{(16)}$.

\section{CONCLUSÃO}

A análise da validade e confiabilidade do MDS adaptado em uma amostra de enfermeiros indicou que o instrumento é válido e fidedigno para avaliar o SM. A análise fatorial do instrumento permitiu o agrupamento das questões em três fatores: falta de competência na equipe de trabalho, negação do papel da enfermagem como advogada do paciente e condições de trabalho insuficientes.

Com a comparação dos dados com outros estudos realizados, os fatores "condições de trabalho insuficientes" e "falta de competência na equipe de trabalho" se mantiveram presentes, demonstrando sua relação com o SM em enfermeiros. O instrumento utilizado mostrou-se confiável, apesar da exclusão de algumas questões em cada nova análise fatorial.

Constatou-se que a falta de competência na equipe de trabalho apresentou maior média e mediana (4,15 e 4,80, respectivamente), demonstrando SM de nivel moderado a intenso.

Considera-se como limitações do estudo o tamanho limitado da amostra, que não permite generalizações a outros contextos, e a extensão do questionário, que apresentou baixa devolução, mesmo com todas tentativas de busca.

\section{REFERÊNCIAS}

1. Jameton A. Dilemmas of moral distress: moral responsibility and nursing practice. Clinical Issues. 1993; 4(4):542-51.

2. Jameton A. Nursing Practice: The ethical issues. Englewood Cliffs, NJ: Prentice-Hall; 1984.

3. Austin W, Kelecevic J, Goble E,Mekechuk J. An overview of moral distress and the pediatric intensive care team. Nurs Ethics.2009;16(1):57-68.

4. Corley MC, Elswick RK, Gorman M,Clor T. Development and evaluation of moral distress scale. J Adv Nurs. 2001;33(2):250-6.

5. Barlem ELD, Lunardi VL, Lunardi GL, Tomaschewski - Berlin JG, Almeida. AS. Caracteristicas psicométricas da moral distress scale em profissionais de enfermagem brasileiros. Texto contexto enferm [Internet]. 2014 [acesso em 2016 jan 7]. Disponivel em: http://www.scielo.br/pdf/tce/v23n3/ pt_0104-0707-tce-2014000060013.pdf.

6. Corley MC, Ptlene M. Elswick RK, Jacob M. Nurse moral distress and ethical work environment. Nurs Ethics. 2005; 12(4):381-90.

7. Hill MM, Hill A. Investigação por questionário. Lisboa: Editora Silado; 2002.

8. Dancey CP, Reidy J. Estatística sem Matemática para Psicologia: usando SPSS para Windows. Porto Alegre: Artmed; 2006.

86 | Enferm. Foco 2016; 7 (1): 81-86
9. Oguisso T, Schmidt MJ. O exercicio da enfermagem: uma abordagem ético-legal. 3ạed Atual e Amp.Rio de Janeiro: Guanabara Koogan; 2010.

10. Grace PJ. Professional advocacy widening the scope of accountability. Nursing Philosophy. 2001; 2(2): 151-62.

11. Dalmolin GL, Lunardi VL, Lunardi FWD. O sofrimento moral dos profissionais de enfermagem no exercicio da profissão. Rev Enf UERJ. 2009; 17(1):35-40

12. Jameton A. A reflection on moral distress in nursing together with a current application of the concept. Bioethical Inquiry. 2013;10:297-308.

13. Barlem ELD, Lunardi VL, Lunardi GL,Dalmolin GL,Tomaschewski JG. Vivência de sofrimento moral na enfermagem: percepção da enfermeira. Rev Esc Enferm USP. 2012;46(3):681-8.

14. Dalmolin GL, Lunardi VL, Barlem ELD, Silveira RS. Implicações do sofrimento moral para os(as) enfermeiros(as) e aproximações com o burnout. Texto Contexto Enferm. 2012; 21(1):200-8.

15. Zuzelo RP. Exploring the Moral Distress of Registered Nurses. Nurs Ethics.2007; 14 (3):343-59.

16. Woods M. Beyond moral distress: Preserving the ethical integrity of nurses. Nurs Ethics. 2014; 21(2):127-8. 